\title{
ON THE DERIVATIVES OF THE LEMPERT FUNCTIONS
}

\author{
NIKOLAI NIKOLOV AND PETER PFLUG
}

\begin{abstract}
We show that if the Kobayashi-Royden metric of a complex manifold is continuous and positive at a given point and any non-zero tangent vector, then the "derivatives" of the higher order Lempert functions exist and equal the respective Kobayashi metrics at the point. It is a generalization of a result by M. Kobayashi for taut manifolds.
\end{abstract}

\section{INTRODUCTION AND RESULTS}

Let $\mathbb{D} \subset \mathbb{C}$ be the unit disc. Let $M$ be an $n$-dimensional complex manifold. Recall first the definitions of the Lempert function $\tilde{k}_{M}$ and the Kobayashi-Royden pseudometric $\kappa_{M}$ of $M$ :

$$
\begin{aligned}
\tilde{k}_{M}^{*}(z, w) & =\inf \{|\alpha|: \exists f \in \mathcal{O}(\mathbb{D}, M): f(0)=z, f(\alpha)=w\}, \\
\tilde{k}_{M} & =\tanh ^{-1} \tilde{k}_{M}^{*}, \\
\kappa_{M}(z ; X) & =\inf \left\{|\alpha|: \exists f \in \mathcal{O}(\mathbb{D}, M): f(0)=z, \alpha f_{*}(d / d \zeta)=X\right\},
\end{aligned}
$$

where $X$ is a complex tangent vector to $M$ at $z$. Note that such an $f$ always exists (cf. [12]; according to [2], page 49, this was already known by J. Globevnik).

The Kobayashi pseudodistance $k_{M}$ can be defined as the largest pseudodistance bounded by $\tilde{k}_{M}$. Note that if $k_{M}^{(m)}$ denotes the $m$-th Lempert function of $M, m \in \mathbb{N}$, that is,

$$
k_{M}^{(m)}(z, w)=\inf \left\{\sum_{j=1}^{m} \tilde{k}_{M}\left(z_{j-1}, z_{j}\right): z_{0}, \ldots, z_{m} \in M, z_{0}=z, z_{m}=w\right\}
$$

then

$$
k_{M}(z, w)=k_{M}^{(\infty)}:=\inf _{m} k_{M}^{(m)}(z, w) .
$$

2000 Mathematics Subject Classification. 32F45.

Key words and phrases. Lempert functions, Kobayashi pseudodistance, Kobayashi-Royden pseudometric, Kobayashi-Buseman pseudometric.

This note was written during the stay of the first named author at the Universität Oldenburg supported by a grant from the DFG, Az. PF 227/8-2 (November December 2006). He likes to thank both institutions for their support. 
By a result of M.-Y. Pang (see [9]), the Kobayashi-Royden metric is the "derivative" of the Lempert function for taut domains in $\mathbb{C}^{n}$; more precisely, if $D \subset \mathbb{C}^{n}$ is a taut domain, then

$$
\kappa_{D}(z ; X)=\lim _{t \rightarrow 0} \frac{\tilde{k}_{D}(z, z+t X)}{t} .
$$

In [6], S. Kobayashi introduces a new invariant pseudometric, called the Kobayashi-Buseman pseudometric in [3]. One of the equivalent ways to define the Kobayashi-Buseman pseudometric $\hat{\kappa}_{M}$ of $M$ is just to set $\hat{\kappa}_{M}(z ; \cdot)$ to be largest pseudonorm bounded by $\kappa_{M}(z ; \cdot)$. Recall that

$$
\hat{\kappa}_{M}(z ; X)=\inf \left\{\sum_{j=1}^{m} \kappa_{M}\left(z ; X_{j}\right): m \in \mathbb{N}, \sum_{j=1}^{m} X_{j}=X\right\}
$$

Thus it is natural to consider the new function $\kappa_{M}^{(m)}, m \in \mathbb{N}$, namely,

$$
\kappa_{M}^{(m)}(z ; X)=\inf \left\{\sum_{j=1}^{m} \kappa_{M}\left(z ; X_{j}\right): \sum_{j=1}^{m} X_{j}=X\right\} .
$$

We call $\kappa_{M}^{(m)}$ the $m$-th Kobayashi pseudometric of $D$. It is clear that $\kappa_{M}^{(m)} \geq \kappa_{M}^{(m+1)}$ and if $\kappa_{M}^{(m)}(z ; \cdot)=\kappa_{M}^{(m+1)}(z ; \cdot)$ for some $m$, then $\kappa_{M}^{(m)}(z ; \cdot)$ $=\kappa_{D}^{(j)}(z ; \cdot)$ for any $j>m$. It is shown in [8] that $\kappa_{M}^{(2 n-1)}=\kappa_{M}^{(\infty)}:=\hat{\kappa}_{M}$, and $2 n-1$ is the optimal number, in general.

We point out that all the introduced objects are upper semicontinuous. Recall that this is true for $\kappa_{M}$ (cf. [7]). It remains to check this for $\tilde{k}_{M}$. We shall use a standard reasoning. Fix $r \in(0,1)$ and $z, w \in M$. Let $f \in \mathcal{O}(\mathbb{D}, M), f(0)=z$ and $f(\alpha)=w$. Then $\tilde{f}=(f, \mathrm{id}): \Delta \rightarrow \tilde{M}=M \times \Delta$ is an embedding. Setting $\tilde{f}_{r}(\zeta)=\tilde{f}(r \zeta)$, by [10], Lemma 3, we may find a Stein neighborhood $S \subset \tilde{M}$ of $\tilde{f}_{r}(\mathbb{D})$. Embed $S$ as a closed complex manifold in some $\mathbb{C}^{N}$ and denote by $\psi$ the respective embedding. Moreover, there is an open neighborhood $V \subset \mathbb{C}^{N}$ of $\psi(S)$ and a holomorphic retraction $\theta: V \rightarrow \psi(S)$. Then, for $z^{\prime}$ near $z$ and $w^{\prime}$ near $w$, we may find, as usual, $g \in \mathcal{O}(\mathbb{D}, V)$ such that $g(0)=\psi\left(z^{\prime}, 0\right)$ and $g(\alpha / r)=\psi\left(w^{\prime}, \alpha\right)$. Denote by $\pi$ the natural projection of $\tilde{M}$ onto M. Then $h=\pi \circ \psi^{-1} \circ \theta \circ g \in \mathcal{O}(\mathbb{D}, M)$, $h(0)=z^{\prime}$ and $h(\alpha / r)=w^{\prime}$. So $r \tilde{k}_{M}^{*}\left(z^{\prime}, w^{\prime}\right) \leq \alpha$, which implies that $\limsup _{z^{\prime} \rightarrow z, w^{\prime} \rightarrow w} \tilde{k}_{M}\left(z^{\prime}, w^{\prime}\right) \leq \tilde{k}_{M}(z, w)$.

To extend Pang's result on manifolds, we have to define the "derivatives" of $k_{M}^{(m)}, m \in \mathbb{N}^{*}=\mathbb{N} \cup\{\infty\}$. Let $(U, \varphi)$ be a holomorphic chart 
near $z$. Set

$$
\mathcal{D} k_{M}^{(m)}(z ; X)=\limsup _{t \rightarrow 0, w \rightarrow z, Y \rightarrow \varphi_{*} X} \frac{k_{M}^{(m)}\left(w, \varphi^{-1}(\varphi(w)+t Y)\right)}{|t|} .
$$

Note that this notion does not depend on the chart used in the definition and

$$
\mathcal{D} k_{M}^{(m)}(z ; \lambda X)=|\lambda| \mathcal{D} k_{M}^{(m)}(z ; X), \quad \lambda \in \mathbb{C} .
$$

Replacing limsup by liminf, we define $\underline{\mathcal{D}} k_{M}^{(m)}$.

From M. Kobayashi's paper [5] it follows that, if $M$ is a taut manifold, then

$$
\hat{\kappa}_{M}(z ; X)=\mathcal{D} k_{M}(z ; X)=\underline{\mathcal{D}} k_{M}(z ; X),
$$

that is, the Kobayashi-Buseman metric is the "derivative" of the Kobayashi distance. The proof there also leads to

$$
\kappa_{M}^{(m)}(z ; X)=\mathcal{D} k_{M}^{(m)}(z ; X)=\underline{\mathcal{D}} k_{M}^{(m)}(z ; X), \quad m \in \mathbb{N}^{*} .
$$

We say that a complex manifold $M$ is hyperbolic at $z$ if $k_{M}(z, w)>$ 0 for any $w \neq z$. We point out that the following conditions are equivalent:

(i) $\mathrm{M}$ is hyperbolic at $z$;

(ii) $\liminf _{z^{\prime} \rightarrow z, w \in M \backslash U} \tilde{k}_{M}\left(z^{\prime}, w\right)>0$ for any neighborhood $U$ of $z$;

(iii) $\underline{\kappa}_{M}(z ; X):=\liminf _{z^{\prime} \rightarrow z, X^{\prime} \rightarrow X} \kappa_{M}\left(z^{\prime} ; X^{\prime}\right)>0$ for any $X \neq 0$;

The implication (i) $\Rightarrow$ (ii) $\Rightarrow$ (iii) are almost trivial (cf. [3]) and the implication (iii) $\Rightarrow$ (i) is a consequence of the fact that $k_{M}$ is the integrated form of $\kappa_{M}$.

In particular, if $M$ is hyperbolic at $z$, then it is hyperbolic at any $z^{\prime}$ near $z$.

Since if $M$ is taut, then it is k-hyperbolic and $\kappa_{M}$ is a continuous function, the following theorem is a generalization of $(*)$.

Theorem 1. Let $M$ be a complex manifold and $z \in M$.

(i) If $M$ is hyperbolic at $z$ and $\kappa_{M}$ is continuous at $(z, X)$, then

$$
\kappa_{M}(z ; X)=\mathcal{D} \tilde{k}_{M}(z ; X)=\underline{\mathcal{D}} \tilde{k}_{M}(z ; X) .
$$

(ii) If $\kappa_{M}$ is continuous and positive at $(z, X)$ for any $X \neq 0$, then

$$
\kappa_{M}^{(m)}(z ; \cdot)=\mathcal{D} k_{M}^{(m)}(z ; \cdot)=\underline{\mathcal{D}} k_{M}^{(m)}(z ; \cdot), \quad m \in \mathbb{N}^{*} .
$$

The first step in the proof of Theorem 1 is the following

Proposition 2. For any complex manifold $M$ one has that

$$
\kappa_{M}^{(m)} \geq \mathcal{D} k_{M}^{(m)}, \quad m \in \mathbb{N}^{*} .
$$


Note that when $M$ is a domain, a weaker version of Proposition 2 can be found in [3], namely, $\hat{\kappa}_{M} \geq \mathcal{D} k_{M}$ (the proof is based on the fact that $\mathcal{D} k_{M}(z ; \cdot)$ is a pseudonorm).

\section{EXAMPLES}

The following examples show that the assumption on continuity in Theorem 1 is essential.

- Let $A$ be a countable dense subset of $\mathbb{C}_{*}$. In [1] (see also [3]), a pseudoconvex domain $D$ in $\mathbb{C}^{2}$ is constructed such that:

(a) $(\mathbb{C} \times\{0\}) \cup(A \times \mathbb{C}) \subset D$;

(b) if $z_{0}=(0, t) \in D, t \neq 0$, then $\kappa_{D}\left(z_{0} ; X\right) \geq C\|X\|$ for some $C=C_{t}>0$. (One can be shown that even $\mathcal{D} \tilde{k}_{D}\left(z_{0} ; X\right) \geq C\|X\|$.)

Then it is easy to see that $\underline{\kappa}_{D}\left(\cdot ; e_{2}\right)=\mathcal{D} k_{D}^{(3)}\left(\cdot ; e_{2}\right)=k_{D}^{(5)}=0$ and $\hat{\kappa}_{D}\left(z_{0} ; X\right) \geq c\|X\|$, where $e_{2}=(0,1)$ and $c>0$. Thus

$$
\hat{\kappa}_{D}\left(z_{0} ; X\right)>\underline{\kappa}_{D}\left(z_{0} ; e_{2}\right)=\mathcal{D} k_{D}^{(3)}\left(z_{0} ; e_{2}\right)=\mathcal{D} k_{D}^{(5)}\left(z_{0} ; X\right), \quad X \neq 0 .
$$

This phenomena obviously extends to $\mathbb{C}^{n}, n>2$ (by considering $\left.D \times \mathbb{D}^{n-2}\right)$. So the inequalities in Proposition 2 are strict in general.

- If $D$ is a pseudoconvex balanced domain with Minkowski function $h_{D}$, then (cf. [3])

$$
h_{D}=\kappa_{D}(0 ; \cdot)=\mathcal{D} \tilde{k}_{D}(0 ; \cdot) .
$$

Therefore, $\mathcal{D} \tilde{k}_{D}(0 ; X)>\underline{\mathcal{D}} \tilde{k}_{D}(0 ; X)$ if $\kappa_{D}(0 ; \cdot)$ is not continuous at $X$. On the other hand, if $\hat{D}$ denotes the convex hull of $D$, then

$$
h_{\hat{D}}=\hat{\kappa}_{D}(0 ; \cdot)=\mathcal{D} k_{D}(0 ; \cdot)=\underline{\mathcal{D}} k_{D}(0 ; \cdot)=\underline{\hat{\kappa}}_{D}(0 ; \cdot) .
$$

- Modifying the first example leads to a pseudoconvex domain $D \subset \mathbb{C}^{2}$ with

$$
L_{\mathcal{D} k_{D}}(\gamma)>0=L_{k_{D}}(\gamma)=L_{\underline{\mathcal{D}} k_{D}}(\gamma),
$$

where $\gamma:[0,1] \rightarrow \mathbb{C}^{2}, \gamma(t):=(t i / 2,1 / 2)$, and $L_{\bullet}(\gamma)$ denotes the respective length.

Indeed, choose a dense sequence $\left(r_{j}\right)$ in $[0, i / 2]$. Put

$$
u(\lambda)=\sum_{k=1}^{\infty} \frac{1}{k^{2}} \log \frac{|\lambda-1 / k|}{4}, \quad v(\lambda)=\sum_{j=1}^{\infty} \frac{u\left(\lambda / 2-r_{j}\right)}{2 j^{2}}, \quad \lambda \in \mathbb{C},
$$

and

$$
D=\left\{z \in \mathbb{C}^{2}: \psi(z)=\left|z_{2}\right| e^{\|z\|^{2}+v\left(z_{1}\right)}<1\right\} .
$$

It is easy to see that $v$ is a subharmonic function on $\mathbb{C}$. Hence $D$ is a pseudoconvex domain with $(\mathbb{C} \times\{0\}) \cup\left(\bigcup_{j, k=1}^{\infty}\left\{r_{j}+1 / k\right\} \times \mathbb{C}\right) \subset D$. 
Observe that $\left.u\right|_{\mathbb{D}}<-1$ and so $D$ contains the unit ball $\mathbb{B}_{2}$. Note also that

$$
k_{D}(a, b)=0, \quad a, b \in \gamma([0,1]) .
$$

Set $\widehat{\psi}(z)=\|z\|^{2} / 2-\log \psi(z)$. Fix $z^{0} \in \mathbb{B}_{2}$ with $\operatorname{Re} z_{1}^{0} \leq 0, \operatorname{Im} z_{2}^{0} \geq 1 / e$. Since $u(\lambda) \geq u(0)$ for $\operatorname{Re} \lambda \leq 0$, we have

$$
\left\|z^{0}\right\| / 2<\widehat{\psi}\left(z^{0}\right)<1-u(0)=: 8 C .
$$

Let $\varphi \in \mathcal{O}(\mathbb{D}, D), \varphi(0)=z^{0}$. Following the estimates in the proof of Example 3.5.10 in [3], we see that $\left\|\varphi^{\prime}(0)\right\|<C$. Hence, $\kappa_{D}\left(z^{0} ; X\right) \geq$ $C\|X\|, X \in \mathbb{C}^{2}$. Since $k_{D}$ is the integrated form of $\kappa_{D}$, it follows that

$$
k_{D}\left(a, a-t e_{1}\right) \geq C t, \quad a \in \gamma([0,1]), 0 \leq t \leq 1 / 2-1 / e, e_{1}=(1,0) .
$$

Hence $\mathcal{D} k_{D}\left(a ; e_{1}\right) \geq C$ and therefore, $L_{\mathcal{D} k_{D}}(\gamma) \geq C / 2>0$, which completes the proof of this example.

Note that it shows that, with respect to the lengths of curves, $\mathcal{D} k_{D}$ behaves different than the "real" derivative of $k_{D}$ (cf. [11] or [4], page 12). Moreover, it implies that, in general, $\mathcal{D} k_{D} \neq \underline{\mathcal{D}} k_{D}$.

Questions. It will be interesting to know examples showing that, in general, $\kappa_{D} \neq \mathcal{D} \tilde{k}_{D}$. It remains also unclear whether $\mathcal{D} k_{D}$ is holomorphically contractible (see [3]). Recall that $\int \mathcal{D} k_{D}=k_{D}$; but we do not know if $\int \underline{\mathcal{D}} k_{D}=k_{D}$.

\section{Proofs}

Proof of Proposition 2. First, we shall consider the case $m=1$. The key is the following

Theorem 3. * [10] Let $M$ be an n-dimensional complex manifold and $f \in \mathcal{O}(\mathbb{D}, M)$ regular at 0 . Let $r \in(0,1)$ and $D_{r}=r \mathbb{D} \times \mathbb{D}^{n-1}$. Then there exists $F \in \mathcal{O}\left(D_{r}, M\right)$, which is regular at 0 and $\left.F\right|_{r \mathbb{D} \times\{0\}}=f$.

Since $\kappa_{M}(z ; 0)=\mathcal{D} \tilde{k}_{M}(z ; 0)=0$, we may assume that $X \neq 0$. Let $\alpha>0$ and $f \in \mathcal{O}(\mathbb{D}, M)$ be such that $f(0)=z$ and $\alpha f_{*}(d / d \zeta)=X$. Let $r \in(0,1)$ and $F$ as in Theorem 3 . Since $F$ is regular at 0 , there exist open neighborhoods $U=U(z) \subset M$ and $V=V(0) \subset D_{r}$ such that $\left.F\right|_{V}: V \rightarrow U$ is biholomorphic. Hence $(U, \varphi)$ with $\varphi=\left(\left.F\right|_{V}\right)^{-1}$, is a chart near $z$. Note that $\varphi_{*}(X)=\alpha e_{1}$, where $e_{1}=(1,0, \ldots, 0)$.

If $w$ and $Y$ are sufficiently near $z$ and $\alpha e_{1}$, respectively, then

$$
g(\zeta):=F(\varphi(w)+\zeta Y / \alpha), \quad \zeta \in r^{2} \mathbb{D},
$$

*We may replace Theorem 3 by the approach used in the proof of the upper semicontinuity of $\tilde{k}_{M}$. 
belongs to $\mathcal{O}\left(r^{2} \mathbb{D}, M\right)$ with $g(0)=w$ and $g(t \alpha)=\varphi^{-1}(\varphi(w)+t Y), t<$ $r^{2} / \alpha$. Therefore, $r^{2} \widetilde{k}_{M}^{*}\left(w, \varphi^{-1}(\varphi(w)+t Y)\right) \leq t \alpha$. Hence $r^{2} \mathcal{D} \tilde{k}_{M}(z ; X) \leq$ $\alpha$. Letting $r \rightarrow 1$ and $\alpha \rightarrow \kappa_{M}(z ; X)$ we get that $\mathcal{D} \tilde{k}_{M}(z ; X) \leq$ $\kappa_{M}(z ; X)$.

Let now $m \in \mathbb{N}$. By definition, $\kappa_{M}^{(m)}(z ; \cdot)$ is the largest function with the following property:

For any $X=\sum_{j=1}^{m} X_{j}$ one has that $\kappa_{M}^{(m)}(z ; X) \leq \sum_{j=1}^{m} \kappa_{M}\left(z ; X_{j}\right)$.

To prove that $\kappa_{M}^{(m)} \geq \mathcal{D} k_{M}^{(m)}$ it suffices to check that $\mathcal{D} k_{M}^{(m)}(z ; \cdot)$ has the same property. Following the above notation and choosing $Y_{j} \rightarrow \varphi_{*} X_{j}$ with $\sum_{j=1}^{m} Y_{j}=Y$, we set $w_{0}=w$ and $w_{j}=\varphi^{-1}(\varphi(w)+$ $t \sum_{k=1}^{j} Y_{j}$ ). Since

$$
k_{M}^{(m)}\left(w, w_{q}\right) \leq \sum_{j=1}^{m} \tilde{k}_{M}\left(w_{j-1}, w_{j}\right),
$$

it follows by the case $m=1$ that

$$
\mathcal{D} k_{M}^{(m)}(z ; X) \leq \sum_{j=1}^{m} \mathcal{D} k_{M}\left(z ; X_{j}\right) \leq \sum_{j=1}^{m} \kappa_{M}\left(z ; X_{j}\right) .
$$

Finally, let $m=\infty$ and $n=\operatorname{dim} M$. Since $\hat{\kappa}_{M}=\kappa_{M}^{(2 n-1)}$ and $k_{M} \leq$ $k_{M}^{(2 n-1)}$, we get that $\mathcal{D} k_{M} \leq \hat{\kappa}_{M}$ using the case $m=2 n-1$.

Proof of Theorem 1. We may assume that $X \neq 0$. In virtue of Proposition 2, we have to show that

$$
\kappa_{M}^{(m)}(z ; X) \leq \underline{\mathcal{D}} k_{M}^{(m)}(z ; X) .
$$

For simplicity we assume that $M$ is a domain in $\mathbb{C}^{n}$.

(i) Fix a neighborhood $U=U(z) \Subset M$. Applying the hyperbolicity of $M$ at $z$, there are a neighborhood $V=V(z) \subset U$ and a $\delta \in(0,1)$ such that, if $h \in \mathcal{O}(\mathbb{D}, M)$ with $h(0) \in V$, then $h(\delta \mathbb{D}) \subset U$. Hence, by the Cauchy inequalities, $\left\|h^{(k)}(0)\right\| \leq c / \delta^{k}, k \in \mathbb{N}$.

Now choose sequences $M \ni w_{j} \rightarrow z, \mathbb{C}_{*} \ni t_{j} \rightarrow 0$, and $\mathbb{C}^{n} \ni Y_{j} \rightarrow X$ such that

$$
\frac{\widetilde{k}_{M}\left(w_{j}, w_{j}+t_{j} Y_{j}\right)}{\left|t_{j}\right|} \rightarrow \underline{\mathcal{D}} \widetilde{k}_{M}(z ; X) .
$$

There are holomorphic discs $g_{j} \in \mathcal{O}(\mathbb{D}, M)$ and $\beta_{j} \in(0,1)$ with $g_{j}(0)=$ $w_{j}, g_{j}\left(\beta_{j}\right)=w_{j}+t_{j} Y_{j}$, and $\beta_{j} \leq \widetilde{k}_{M}^{*}\left(w_{j}, w_{j}+t_{j} Y_{j}\right)+\left|t_{j}\right| / j$. Note that $\widetilde{k}_{M}^{*}\left(w_{j}, w_{j}+t_{j} Y_{j}\right) \leq c_{1}|| t_{j} Y_{j} \| \leq c_{2}\left|t_{j}\right|$.

Write

$$
w_{j}+t_{j} Y_{j}=g_{j}\left(\beta_{j}\right)=w_{j}+g_{j}^{\prime}(0) \beta_{j}+h_{j}\left(\beta_{j}\right)
$$


Then

$$
\left\|h_{j}\left(\beta_{j}\right)\right\| \leq c \sum_{k=2}^{\infty}\left(\frac{\beta_{j}}{\delta}\right)^{k} \leq c_{3}\left|\beta_{j}\right|^{2} \leq c_{4}\left|t_{j}\right|^{2}, \quad j \geq j_{0} .
$$

Put $\widehat{Y}_{j}=Y_{j}-h_{j}\left(\beta_{j}\right) / t_{j}$. We have that $g_{j}(0)=w_{j}$ and $\beta_{j} g_{j}^{\prime}(0) / t_{j}=$ $\widehat{Y}_{j} \rightarrow X$. Therefore,

$$
\kappa_{M}\left(w_{j} ; \widehat{Y}_{j}\right) \leq \frac{\beta_{j}}{\left|t_{j}\right|} \leq \frac{\widetilde{k}_{M}^{*}\left(z_{j}, w_{j}+t_{j} Y_{j}\right)}{\left|t_{j}\right|}+\frac{1}{j} .
$$

Hence with $j \rightarrow \infty$, we get that $\kappa_{M}(z ; X)=\underline{\kappa}_{M}(z ; X) \leq \underline{\mathcal{D}} \widetilde{k}_{M}(z ; X)$.

(ii) The proof of the case $m \in \mathbb{N}$ is similar to the next one and we omit it. Now, we shall consider the case $m=\infty$.

Note first that our assumption implies that $M$ is hyperbolic at $z$ and, by the contrary,

$$
\begin{aligned}
\forall \varepsilon>0 \exists \delta>0:\|w-z\|<\delta,|| Y-X\|<\delta\| X \| \\
\quad \Rightarrow\left|\kappa_{M}(w ; Y)-\kappa_{M}(z ; X)\right|<\varepsilon \kappa_{M}(z ; X) .
\end{aligned}
$$

Moreover, the proof of (i) shows that

$$
\tilde{k}_{M}(a, b) \geq \kappa_{M}(a ; b-a+o(a, b)), \text { where } \lim _{a, b \rightarrow z} \frac{o(a, b)}{\|a-b\|}=0 .
$$

Choose now sequences $M \ni w_{j} \rightarrow z, \mathbb{C}_{*} \ni t_{j} \rightarrow 0$, and $\mathbb{C}^{n} \ni Y_{j} \rightarrow X$ such that

$$
\frac{k_{M}\left(w_{j}, w_{j}+t_{j} Y_{j}\right)}{\left|t_{j}\right|} \rightarrow \underline{\mathcal{D}} k_{M}(z ; X) .
$$

There are points $w_{j, 0}=w_{j}, \ldots, w_{j, m_{j}}=w_{j}+t_{j} X_{j}$ in $M$ such that

$$
\sum_{k=1}^{m_{j}} \tilde{k}_{M}\left(w_{j, k-1}, w_{j, k}\right) \leq k_{M}\left(w_{j}, w_{j}+t_{j} Y_{j}\right)+\frac{1}{j} .
$$

Set $w_{j, k}=w_{j}$ for $k>m_{j}$. Since

$k_{M}\left(w_{j}, w_{j, l}\right) \leq \sum_{j=1}^{l} \tilde{k}_{M}\left(w_{j, k-1}, w_{j, k}\right) \leq k_{M}\left(w_{j}, w_{j}+t_{j} Y_{j}\right)+\frac{1}{j} \leq c_{2}\left|t_{j}\right|+\frac{1}{j}$,

then $k_{M}\left(w_{j}, w_{j, l}\right) \rightarrow 0$ uniformly in $l$. Then the hyperbolicity of $M$ at $z$ implies that $w_{j, l} \rightarrow z$ uniformly in $l$. Indeed, assuming the contrary and passing to a subsequence, we may suppose that $w_{j, l_{j}} \notin U$ for some $U=U(z)$. Then

$$
0=\lim _{j \rightarrow \infty} k_{M}\left(w_{j}, w_{j, l}\right) \geq \liminf _{z^{\prime} \rightarrow z, w \in M \backslash U} \tilde{k}_{M}\left(z^{\prime}, w\right)>0,
$$

a contradiction. 
Fix now $R>1$. Then (1) implies that

$\kappa_{M}\left(z ; w_{j, k}-w_{j, k-1}\right) \leq R \kappa_{M}\left(w_{j, k} ; w_{j, k}-w_{j, k-1}+o\left(w_{j, k}, w_{j, k-1}\right)\right), j \geq j(R)$.

It follows by this inequality, (2) and (3) that

$$
\sum_{k=1}^{m_{j}} \kappa_{M}\left(z ; w_{j, k}-w_{j, k-1}\right) \leq R k_{M}\left(w_{j}, w_{j}+t_{j} Y j\right)+\frac{R}{j} .
$$

Since $\hat{\kappa}_{M}\left(z ; t_{j} Y_{j}\right)$ is bounded by the first sum, we obtain that

$$
\hat{\kappa}_{M}\left(z ; Y_{j}\right) \leq R \frac{k_{M}\left(w_{j}, w_{j}+t_{j} Y j\right)+1 / j}{\left|t_{j}\right|} .
$$

Note that $\hat{\kappa}_{M}(z ; \cdot)$ is a continuous function. Hence with $j \rightarrow \infty$ and $R \rightarrow 1$, we get that $\hat{\kappa}_{M}(z ; X) \leq \underline{\mathcal{D}} k_{M}(z ; X)$.

Remark. It follows by the above proofs and a standard diagonal process that $\underline{\kappa}_{M}(z ; \cdot)=\underline{\mathcal{D}} \tilde{k}(z ; \cdot)$ if $M$ is hyperbolic at $z$.

\section{REFERENCES}

[1] K. Diederich, N. Sibony, Strange complex structures on Euclidian space, J. Reine Angew. Math. 311/312 (1979), 397-407.

[2] S. Dineen, The Schwarz lemma, Oxford Math. Monographs, Clarendon Press, Oxford, 1989.

[3] M. Jarnicki, P. Pflug, Invariant distances and metrics in complex analysis, de Gruyter Exp. Math. 9, de Gruyter, Berlin, New York, 1993.

[4] M. Jarnicki, P. Pflug, Invariant distances and metrics in complex analysisrevisited, Dissertationes Math. 430 (2005).

[5] M. Kobayashi, On the convexity of the Kobayashi metric on a taut complex manifold, Pacific J. Math. 194 (2000), 117-128.

[6] S. Kobayashi, A new invariant infinitesimal metric, International J. Math. 1 (1990), 83-90.

[7] S. Kobayashi, Hyperbolic complex spaces, Grundlehren Math. Wiss. 318, Springer, Berlin, 1998.

[8] N. Nikolov, P. Pflug, On the definition of the Kobayashi-Buseman pseudometric, International J. Math. (to appear).

[9] M.-Y. Pang, On infinitesimal behavior of the Kobayashi distance, Pacific J. Math. 162 (1994), 121-141.

[10] H.-L. Royden, The extension of regular holomorphic mapps, Proc. Amer. Math. Soc. 43 (1974), 306-310.

[11] S. Venturini, Pseudodistances and pseudometrics on real and complex manifolds, Ann. Mat. Pura Appl. 154 (1989), 385-402.

[12] J. Winkelmann, Non-degenerate maps and sets, Math. Z. 249 (2005), 783-795.

Institute of MATHEMATiCs AND Informatics, Bulgarian ACADEMY OF Sciences, Acad. G. Bonchev 8, 1113 Sofia, Bulgaria

E-mail address: nik@math.bas.bg 
Carl von Ossietzky Universität Oldenburg, Institut für Mathematik, Fakultät V, Postfach 2503, D-26111 Oldenburg, Germany

E-mail address: pflug@mathematik.uni-oldenburg.de 